Sessions d'étude - Société canadienne d'histoire de l'Église catholique

\title{
Le Centre de recherche en histoire religieuse du Canada
}

\section{Pierre Hurtubise}

Volume 38, 1971

La genèse de la Société des Missions-Étrangères de la province de Québec

URI : https://id.erudit.org/iderudit/1007267ar

DOI : https://doi.org/10.7202/1007267ar

Aller au sommaire du numéro

Éditeur(s)

Les Éditions Historia Ecclesiæ Catholicæ Canadensis Inc.

ISSN

0318-6172 (imprimé)

1927-7067 (numérique)

Découvrir la revue

Citer cet article

Hurtubise, P. (1971). Le Centre de recherche en histoire religieuse du Canada. Sessions d'étude - Société canadienne d'histoire de l'Église catholique, 38, 85-88. https://doi.org/10.7202/1007267ar

Tous droits réservés @ Les Éditions Historia Ecclesiæ Catholicæ Canadensis Inc., 1972
Ce document est protégé par la loi sur le droit d'auteur. L'utilisation des services d'Érudit (y compris la reproduction) est assujettie à sa politique d'utilisation que vous pouvez consulter en ligne.

https://apropos.erudit.org/fr/usagers/politique-dutilisation/ 


\section{Table ronde}

Au programme du congrès figurait une discussion autour des objectifs poursuivis et à poursuivre par notre Société. Quatre spécialistes avaient accepté de présenter le point de vue de divers organismes intéressés à l'histoire religieuse. Ce sont: le P. Benoit Lacroix, o.p., M. René Hardy, le P. Pierre Hurtubise, o.m.i., et le P. Adrien Pouliot, s.j. Les trois premiers nous ont autorisés à reproduire leurs propos.

\section{Le Centre de recherche en histoire religieuse du Canada}

Le Centre de recherche en histoire religieuse du Canada se veut surtout centre de recherche et de documentation. Il ne se désintéresse pas de l'aspect promotion et coordination de la recherche $\neg$ la collection qu'il dirige chez Fides de même que les colloques qu'il organise en font foi -, mais il entend tout de même faire porter l'essentiel de ses efforts sur la recherche elle-même et sur la confection des instruments de base de cette recherche. Ainsi travaillons-nous présentement à la mise sur pied d'un fichier bibliographique couvrant tous les aspects de l'histoire religieuse du Canada en même temps que nous entreprenons le dépouillement systématique et, dans la mesure du possible, le microfilmage d'un certain nombre de fonds d'archives européens relatifs à cette même histoire.

C'est à partir de ces préoccupations que nous voudrions répondre à l'invitation qui nous a été faite de dire ce que nous attendons de la Société canadienne d'histoire de l’Église catholique.

Avant que d'y arriver, nous tenons toutefois à souligner, dès l'abord, la contribution très importante apportée depuis 1933 par la SCHEC au développement d'une historiographie religieuse propre au Canada. La seule collection des rapports annuels suffirait à elle seule à montrer de quel poids a été cette contribution, mais il faut encore ajouter à ce total nombre d'articles et d'ouvrages qui ont eu comme point de départ une communication présentée lors d'un congrès de la Société et nombre de curiosité éveillées dans le public participant aussi bien que dans le public lecteur. Ajoutons que depuis quelques années les rapports annuels se sont enrichis de précieuses bibliographies qui rendent déjà de très grands services. C'est justement cet effort que nous voudrions voir poursuivi et, si possible, amplifié.

A l'article second de sa constitution, la SCHEC définit ses objectifs comme suit: 
1) encourager les travaux historiques et stimuler l'intérêt du public pour l'histoire de l'Église;

2) assurer la conservation des lieux historiques, des documents, des reliques et des souvenirs importants du passé;

3) publier des études et des documents historiques, dans la mesure du possible.

Ces objectifs, bien entendu, n'ont pu être jusqu'ici que partiellement réalisés. Qui osera en faire grief à la Société, compte tenu des difficultés et des obstacles qui se sont présentés au fil des années - difficultés financières, entre autres - compte tenu également du caractère ambitieux du programme qu'on s'était imposé ?

Nous ne voudrions d'ailleurs retenir ici que certains aspects de ce programme qui nous paraissent d'une urgence toute particulière et, par conséquent, dignes d'une considération spéciale de la part de la SCHEC.

\section{Inventaire des sources}

En premier lieu, le problème des sources. Dès 1934, la Société avait mis sur pied un comité des archives et de la bibliographie. Dans le rapport annuel qui suivit, MM. Bonin et Caron firent paraître une description sommaire des archives de Saint-Sulpice et de celles de l'archevêché de Québec. Début des plus prometteurs! Malheureusement, il fallut attendre près de trente ans pour en voir la suite, car ce n'est qu'en 1963 et grâce aux soins du P. Gaston Carrière, o.m.i., que le rapport annuel put reprendre cette rubrique sous forme d'une analyse sommaire des fonds d'archives diocésaines.

Il faudrait au plus tôt réactiver et compléter cette entreprise qui n'a été, à vrai dire, jusqu'ici qu'ammorcée. Car il ne s'agit pas en l'occurence que d'archives diocésaines: il s'agit également d'archives paroissiales, d'archives de communautés et d'établissement religieux, d'archives d'associations et de mouvements catholiques (v.g. Action catholique générale et spécialisée, Zouaves, ACJC, syndicats catholiques, etc.), voire même d'archives privées.

A cette fin, la SCHEC devrait

1) reconstituer au plus tôt un comité des archives;

2) encourager les diverses archives religieuses à procéder, si elles ne l'ont déjà fait, à un classement systématique de leurs fonds; les convaincre de mettre ces fonds le plus tôt possible à la disposition des chercheurs et, pour ce faire, leur montrer l'importance de confier ces fonds à un personnel qualifié; 
3) établir une liste aussi complète que possible de ces divers fonds d'archives, en obtenir une description au moins sommaire en vue de la publication d'un guide des archives religieuses du Canada.

Nous tenons à signaler, à ce propos, que le CRHRC a été approché par le représentant d'un certain nombre de groupes protestants qui avait lui-même pris contact avec la direction des Archives nationales du Canada au sujet d'un inventaire national des archives religieuses de toutes dénominations. La SCHEC ne pourrait-elle pas se charger d'assurer la partie catholique de cette enquête, quitte à faire appel à la collaboration des historiens, des archivistes et des autorités intéressés. Ce serait là un projet qui se situerait tout à fait dans la ligne des préoccupations de la SCHEC et qui serait, d'autre part, appelé à rendre les plus grands services.

\section{Les congrès annuels}

Il est un autre point sur lequel nous voudrions insister, c'est celui de l'organisation et de l'orientation des congrès annuels de la SCHEC. Il nous paraît urgent de songer à intégrer dans toute la mesure du possible les travaux présentés lors des congrès dans la trame des recherches actuellement en cours dans les départements et instituts d'histoire de nos universités. A cet effet, il importerait, d'une part, de toujours assigner un thème précis à chaque congrès - politique qui n'a pas toujours été suivie par le passé - et, d'autre part, de ne choisir ce thème qu'après consultation d'un certain nombre de spécialistes au fait de l'état de la recherche en histoire religieuse au Canada. Les congrès de la SCHEC ont beaucoup fait jusqu'ici pour stimuler cette recherche: leur contribution pourrait être beaucoup plus importante et beaucoup plus utile si on voulait bien la placer sous le signe d'objectifs plus précis et mieux délimités.

Ne l'oublions pas: nous vivons à une époque où la coordination des efforts et le travail d'équipe deviennent une nécessité inéluctable à tous les niveaux de la recherche. La SCHEC ne peut échapper à cette nouvelle réalité. $D$ 'ailleurs la situation faite à l'histoire a beaucoup changé depuis 1933. Nous étions encore à l'époque au stade des premiers défrichements. Les spécialistes étaient peu nombreux et les moyens de le devenir, plutôt limités. Telle n'est plus la situation aujourd'hui. Le nombre des départements et instituts d'histoire ne cesse de croître en même temps que celui de nos universités. Chaque région importante en est désormais pourvue et dans chacune d'elles des spécialistes, sinon des équipes de spécialistes poursuivent des recherches sur tous les aspects de l'histoire canadienne, y compris l'aspect religieux. Pourquoi la SCHEC ne deviendrait-elle pas le lieu de rassemblement et de réflexion de tous ceux de ces spécialistes qui s'intéressent à l'histoire de l'Église du Canada, mais également de leurs étudiants et, pourquoi pas, de leurs 
lecteurs, pour justement être mieux à même de répondre à son premier objectif qui est de "susciter l'intérêt pour l'histoire de l'Église "? $\mathrm{Ne}$ serait-il pas à souhaiter que les réunions annuelles deviennent véritablement les États généraux de l'histoire de l'Église canadienne servant à regrouper tous ceux, spécialistes et amateurs, que passionne notre passé religieux ? C'est un vœu que nous osons formuler.

Voilà les suggestions que nous avions à faire: nous prions la direction de la SCHEC de bien vouloir les prendre le plus tôt possible en considération.

Pierre Hurtubise, o.m.i., Université Saint-Paul, Ottawa, Ont.

\section{Un Centre d'études des religions populaires}

Ce n'est pas à une honorable société comme La Société canadienne $d^{\prime}$ Histoire de l'Église catholique qu'on voudrait faire la leçon. Mais la situation religieuse étant devenue ce qu'elle est présentement, au Canada français et plus spécialement au Québec, nous craignons malgré nous que soient à jamais dévalorisés, oubliés même, en même temps qu'écartés de la vie pratique, des rites, des attitudes, des croyances qui ont véhiculé certaines valeurs sans lesquelles notre "petit peuple», comme disait le chanoine Groulx, n'aurait sûrement pas survécu et qui l'ont fait tel, riche en agir, sans qu'il sache toujours pourquoi. Pour sa part le Centre d'études des religions populaires (C.E.R.P.) de l'Université de Montréal n'a d'autres intentions que d'identifier ces phénomènes religieux « populaires », quels qu'ils soient, en rassemblant, préservant et, si possible, conservant le meilleur grâce à des archives écrites et audio-visuelles.

Que je vous explique en premier lieu (I) le contexte dans lequel a été créé et évolue encore le C.E.R.P., (II) les objectifs de l'équipe; pour vous dire enfin (III) les urgences qui présentement retiennent davantage notre attention. Les échanges qui suivront permettront les précisions que cet exposé veut appeler plutôt qu'expliciter.

\section{I \\ Un contexte de religion d'Occident}

Le Centre d'études des religions populaires (C.E.R.P.) existe depuis 1967; il est présentement greffé à une institution de recherches interdisciplinaires, l'Institut d'Études médiévales, qui œuvre à l'Université de Montréal depuis 1942. Cet organisme qui s'est spécialisé surtout dans le champ mixte des études littéraires et religieuses du moyen âge occidental compte près de 50 publications scientifiques éditées conjointement à Montréal et à Paris, chez J. Vrin. C'est donc à dire que toute recherche s'y trouve en lieu sûr, d'autant plus que l'objectif de cet Institut scientifique est l'identification, à ses origines, de l'homme d'Occi- 